Sādhanā Vol. 40, Part 3, May 2015, pp. 1001-1022. (C) Indian Academy of Sciences

\title{
Oscillatory and electrohydrodynamic instabilities in flow over a viscoelastic gel
}

\author{
R M THAOKAR \\ Department of Chemical Engineering, Indian Institute of Technology, \\ Mumbai 400 076, India \\ e-mail: rochish@che.iitb.ac.in
}

MS received 12 September 2014; revised 06 February 2015; accepted 11 February 2015

\begin{abstract}
The stability of oscillatory flows over compliant surfaces is studied analytically and numerically. The type of compliant surfaces studied is the incompressible viscoelastic gel model. The stability is determined using the Floquet analysis, where amplitude of perturbations at time intervals separated by one time period is examined to determine whether perturbations grow or decay. Oscillatory flows pas viscoelastic gels exhibit an instability in the limit of zero Reynolds number, and the transition amplitude of the oscillatory velocity increases with the frequency of oscillations. The transition amplitude has a minimum at a finite wavenumber for the viscoelastic gel model. The instability is found to depend strongly on the gel viscosity $\eta_{g}$, and the effect of oscillations on the continuation of viscous modes at intermediate Reynolds number shows a complicated dependence on the oscillation frequency. Experimental studies are carried out on the stability of an oscillatory flow past a viscoelastic gel at zero Reynolds number, and these confirm the theoretical predictions.
\end{abstract}

Keywords. Oscillatory flows; fluid-structure and electrohydrodynamic instabilities; floquet theory.

\section{Introduction}

Fluid flow over soft materials is ubiquitous in biological as well as in technological applications. These soft materials could be infinitesimally thin viscoelastic membranes, like those occurring in red blood cells, or flexible walls of finite thickness, like the walls of blood vessels. The dynamics of fluid flow past flexible surfaces is qualitatively different from the flow over rigid surfaces, because of the coupling between the fluid and the wall dynamics, and the wall properties can influence the stability characteristics in a non-trivial manner. Oscillatory flow over soft materials is encountered in the flow of cardiovascular fluids through flexible blood vessels which are driven by the pumping of the heart. The Reynolds numbers for these flows vary over a wide range between $\operatorname{Re}<1$ and $\operatorname{Re}=4000(\mathrm{Ku} 1997)$. The oscillatory nature of the blood flow in the vascular system is characterized by a number often called the Womersley or Witzig number 
$\alpha=R \sqrt{\frac{\omega}{v}}$, where $R$ is the artery diameter, $\omega$ is the oscillatory frequency and $v$ is the kinematic viscosity of the cardiovascular fluid, and these arterial flows can be affected by the wall elasticity and conditions like stenosis, when the arteries get narrowed and obstructed. There has been a great deal of experimental and computational fluid dynamics studies to calculate the oscillatory and peristaltic fluid flow through elastic tubes. The elasticity of the wall is carefully accounted for in the analysis, and is found to affect the flow in a significant manner. However, all these studies are carried out for fluid flow through tubes or surfaces which, although flexible, are of fixed geometries. Not much work has been done on the moving boundary elastic solid-fluid interaction problems, and so the problem of onset of instability and full numerical evolution of perturbations has not been addressed till date.

The objective of the study of the stability of steady flows is to determine whether the growth rate of perturbations in time is positive or negative. It is difficult to use a similar procedure for oscillatory flows, since the mean velocity is a periodic function of time. If the growth rate is large compared to the frequency of oscillations, it is possible to approximate the flow at any instant of time as a steady flow with the instantaneous mean velocity, and examine the growth of perturbations for this flow. In this case, it is expected that if the velocity at any point in the oscillatory cycle is greater than the critical velocity for the steady flow, perturbations will grow rapidly in a time scale short compared to the period of oscillation and take the system into the non-linear regime. However, if the growth rate is not large compared to the frequency of oscillations, the growth of perturbations during the part of the cycle when the velocity is greater than the critical velocity for a steady flow could be compensated by the decay of perturbations during the part of the cycle when the velocity is lower than that for a steady flow. In this case, it is necessary to examine the relative amplitude of perturbations at equal phase angles in successive cycles, in order to determine whether there is growth or decay of perturbations as the cycles progress. This is carried out in the present study using the Floquet analysis.

The stability of a horizontal liquid layer on an oscillating plate was studied by Yih (1968) using low wavenumber asymptotic analysis. The oscillatory flow was shown to be unstable, although the non-oscillatory flow is stable. Von Kerckzek \& Davis (1974) studied the flow of a layer of fluid down an inclined oscillating plane. This system is known to be unstable in the absence of oscillation. Von Kerckzek \& Davis (1974) has calculated the stability windows for this flow in the presence of oscillations. The analysis of Von Kerckzek \& Davis (1974) indicates that although the Stokes layer has velocity profiles with inflection points, those are unstable only at low frequencies. At sufficiently high frequencies, the inflectional unstable modes may not have enough time to grow, and can be eventually stabilized by the rapidly oscillating flow. Coward \& Papageorgiou (1994) studied the stability of two-phase Couette flow bounded between plane parallel plates using the long wave analysis of Yih, where one of the bounded plates had a time dependent velocity in its plane. The time dependent velocity had a constant and an oscillatory time periodic part. The flow is unstable in the nonoscillatory regime due to an interfacial instability if the viscosities are different. Using Floquet theory, the authors showed that in the long wave limit, the time modulations can have significant influence on flow stability. Their analysis showed that flows which are otherwise unstable can be stabilized by oscillations. King et al (1999) studied the problem of oscillatory two-phase Couette flow experimentally as well as numerically. Since the Floquet theory gives only time averaged growth rates, they calculated the transient instability numerically and showed that the interfacial wave amplification actually originates with an internal disturbance, and is not directly caused by interfacial shear. Coward \& Renardy (1997) studied the effect of oscillatory forcing as a dynamic stabilisation mechanism for two-layer plane Couette-Poiseuille flow at low Reynolds numbers using numerical and 
asymptotic methods. They found that the oscillations can have stabilising or destabilizing effect depending upon the conditions of the flow. Complete stabilisation is possible for certain flows which are otherwise unstable owing to the viscosity stratification across the interface. The combined pressure and velocity fluctuations can have an effect on the flow stability opposite to that induced by the time-periodic forcing mechanisms. There has been very little work on the stability of viscoelastic oscillatory flows. Ramanan et al (1999) studied the stability of viscoelastic shear flows subjected to steady and oscillatory transverse flows. Their study showed that superposition of axial periodic motion on circular Couette and Dean flows can delay the onset of the viscoelastic instability. However, there does not seem to have been much work done on the stability of oscillatory fluid flows over compliant surfaces, which forms the basis of the present work.

Oscillatory flows are unsteady, and it is important to precisely define the stability of an unsteady flow. Presented below is the description of stability in unsteady flows as discussed in Davis (1976). In oscillatory flows, since the base state itself is unsteady, a natural measure of the system stability would be the comparison between the growth rate of the system and the rate of change of the base state. However difficulties arise in interpretation, since the time periodic base state accelerates in the first cycle and decelerates in the second half. A system would be called monotonically stable if the disturbances decay at every point in time. However there can be cases in which the disturbances grow in the first half of the cycle and then decay, so that there is a net decay of the perturbation over the cycle. These are called transiently stable systems. However, the perturbation can attain sufficient amplitude during the growth cycle, so that the perturbation amplitude is no longer small compared to the mean flow velocity. In this case, nonlinear interactions can then lead to secondary flows. This is a more difficult mathematical problem, and in the present analysis, we assume that the amplitude of the disturbances remain small at all times over the cycle, so that the non-linear terms can be neglected.

Oscillatory systems are characterised by an additional time scale in the system, the oscillation frequency, which also gives an additional length scale, called the Stokes length $(v / \omega)^{1 / 2}$. The perturbations due to plate oscillations result in velocity disturbances over length scales comparable to the Stokes length. When the Stokes length is small compared to the width of the channel, the flow resembles a boundary layer flow where disturbances are localized near the oscillating plate, and it is expected that interfacial instabilities will not be affected by this type of forcing. The most interesting regime is when the Stokes length is comparable to the width of the channel, when there is a coupling between the motion of the top plate and the interfacial motion. This regime is examined in the present analysis.

An interfacial electrohydrodynamic instability due to difference between the electrical properties of two fluids is well investigated in the literature (Gambhire \& Thaokar 2012 and references therein). In the present work, linear and weakly non-linear analyses are carried out for the electrohydrodynamic instability of a fluid-gel system, which consists of a fluid flowing past a viscoelastic solid in the zero Reynolds number limit. In the latter case, it is known that there is a linear instability induced by flow in the limit of zero Reynolds number, and it is of interest to study the effect of flow on the electrodynamic instability, and the effect of the electric field on the viscous instability.

The article is organised in the following manner: the Floquet theory of dynamical equations is discussed in section 2. The oscillatory flow instability over viscoelastic gels is analyzed in section 3. The experimental results for the low Reynolds number instability in flow over viscoelastic gels are presented in section 4 . The results of electrohydrodynamic instability in flow over a viscoelastic gel are described in section 5 . 


\section{Floquet theory for the dynamical equations}

The Floquet analysis is used to determine the stability of time periodic base states or limit cycles. A Floquet exponent larger than one implies that the limit cycle is unstable to small perturbations. The relation between the Floquet exponents and the characteristic root, which is the growth rate in the linear analysis for a non-oscillatory flow, is as follows. Consider a linear dynamical system governed by the equation

$$
\dot{\mathbf{x}}=\mathbf{L x}
$$

where $\mathbf{x}$ is a column vector, the superscript ${ }^{*}$ represents the time derivative, and $\mathbf{L}$ is a matrix with periodic coefficients which have time period $T$. If $\mathbf{x}_{0}(t)$ is a solution of Eq. 1 , it is easy to see that if

$$
\mathbf{x}_{0}(t+(n+1) T)=\mathbf{A} \mathbf{x}_{0}(t+n T)
$$

where $n$ is an integer, then

$$
\mathbf{A}=\exp \left(\int_{t}^{t+T} d t^{\prime} \mathbf{L}\left(t^{\prime}\right)\right)=\exp (\mathbf{B}),
$$

where $B$ is a square matrix. In addition, it can be easily inferred that if all eigenvalues of $\mathbf{A}$ are discrete and $\mathbf{A}$ is non-singular so that it can be reduced to its Jordan form, the eigenvectors of $\mathbf{B}$ are identical to the eigenvectors of $\mathbf{A}$, and $\alpha_{i}$, the eigenvalues of $\mathbf{A}$, are related to $\theta_{i}$, the eigenvalues of $\mathbf{B}$, by

$$
\alpha_{i}=\exp \left(\theta_{i}\right)
$$

The eigen values of $\mathbf{A}$ determine whether the components of the vector $\mathbf{x}_{0}$ decay or grow as time progresses. If any eigenvalue of $\mathbf{A}$ is greater than 1 (or any eigenvalue of $\mathbf{B}$ is positive), then the projection of $\mathbf{x}_{0}$ in the direction of the corresponding eigenvector of $\mathbf{A}$ increases as the number of time periods increases, whereas if all eigenvalues $\mathbf{A}$ are less than 1 (or all eigenvalues of $\mathbf{B}$ have negative real parts), then all components of $\mathbf{x}_{0}$ decay in time. For a steady flow in which $\mathbf{L}$ is independent of time, the eigenvalues $\theta_{i}$ of $\mathbf{B}$ are related to the eigenvalues $\lambda_{i}$ of $L$ (the linear growth rates) by

$$
\theta_{i}=\frac{\lambda_{i}}{T}
$$

The stability of an oscillatory flow which satisfies the equation

$$
\dot{\mathbf{x}}(t)=F(\mathbf{x}(t))
$$

is determined as follows. First, the time periodic solution $\overline{\mathbf{x}}$ which satisfies the Eq. 6 is identified. The solution is then perturbed about this state using a perturbation of the form $\mathbf{x}=\overline{\mathbf{x}}+\mathbf{x}^{\prime}$, and Eq. 6 is linearized in the amplitude of the perturbations $\mathbf{x}^{\prime}$ to obtain an equation of the form

$$
\dot{\mathbf{x}}^{\prime}=\mathbf{L} \dot{\mathbf{x}}^{\prime}
$$

Equation 7 is then reduced to an equation of the form 2, and the eigenvalues of the matrix $\mathbf{A}$ (or equivalently the Floquet exponents, which are the eigenvalues $\theta_{i}$ of the matrix $\mathbf{B}$ ) are extracted to determine the stability of the time periodic state. In the present work, we discuss the stability characteristics in terms of the eigenvalue $\theta_{i}$, the growth rate of the system. The system is stable if the growth rate $\theta_{i}$ is negative and unstable if $\theta_{i}$ is positive. 


\section{Oscillatory flow over viscoelastic gels}

The configuration consists of an incompressible viscoelastic gel of thickness $H R^{*}$ grafted to a rigid surface, and an incompressible Newtonian fluid layer of thickness $R^{*}$ between the gel surface and a rigid top plate. In this case, the material parameters that affect the dynamics of the system are the density of the fluid and gel $\rho^{*}$ (assumed to be equal), the shear modulus $E^{*}$ and viscosity $\mu_{g}^{*}$ of the gel, in addition to the fluid viscosity $\mu^{*}$. The velocity is scaled by $\left(E^{*} R^{*} / \mu^{*}\right)$, the length by $\left(R^{*}\right)$, time by $\left(\mu^{*} / E^{*}\right)$, the pressure by $\left(E^{*}\right)$, and the relative viscosity $\eta_{g}=\left(\mu_{g}^{*} / \mu^{*}\right)$ is defined as the ratio of the viscosity of the gel and fluid. The nondimensional top velocity is then given by $\Lambda+A \cos (\Omega t)$, where the non-dimensional strain rates are $\Lambda=\left(V^{*} \mu^{*} / E^{*} R^{*}\right)$ and $A=\left(A^{*} \mu^{*} / E^{*} R^{*}\right)$.

The scaled equations for the fluid are

$$
\begin{aligned}
\nabla \cdot \mathbf{v} & =0 \\
\Sigma_{g}\left(\partial_{t} \mathbf{v}+\mathbf{v} \cdot \nabla \mathbf{v}\right) & =-\nabla P^{f}+\nabla^{2} \mathbf{v}
\end{aligned}
$$

where $\Sigma_{g}=\frac{R e}{\Lambda}=\frac{R e_{0}}{\Lambda}=\frac{\rho^{*} R^{* 2} E^{*}}{\mu^{* 2}}, R e=\frac{\rho^{*} V^{*} H^{*}}{\mu^{*}}$, and $R e_{o}=\frac{\rho^{*} A^{*} H^{*}}{\mu^{*}}$ are the steady and oscillatory Reynolds numbers. The equations for the displacement field in the gel, u, which represents the displacement of material points from their steady state positions due to the stresses exerted on the gel, are

$$
\begin{aligned}
\nabla . \mathbf{u} & =0 \\
\Sigma_{g} \partial_{t}^{2} \mathbf{u} & =-\nabla P^{g}+\left(1+\eta_{g} \partial_{t}\right) \nabla^{2} \mathbf{u} .
\end{aligned}
$$

The base state velocity profile is governed by the equation

$$
\Sigma_{g} \frac{\partial \bar{V}_{x}}{\partial t}=\frac{\partial^{2} \bar{V}_{x}}{\partial z^{2}}
$$

and the governing equation for the mean gel displacement is given by

$$
\Sigma_{g} \frac{\partial^{2} \bar{U}_{x}}{\partial t^{2}}=\left(1+\eta_{g} \frac{\partial}{\partial t}\right) \frac{\partial^{2} \bar{U}_{x}}{\partial z^{2}} .
$$

where $\mathbf{u}$ is the displacement field, which represents the displacement of material points from their steady state positions due to stresses exerted on the gel. The boundary conditions are

$$
\begin{aligned}
\left.\bar{V}_{x}\right|_{z=1} & =\Lambda+A \cos (\Omega t) \\
\left.\bar{V}_{x}\right|_{z=0} & =\left.\frac{d \bar{U}_{x}}{d t}\right|_{z=0} \\
\left.\frac{d \bar{V}_{x}}{d z}\right|_{z=0} & =\left.\left(1+\eta_{g} \frac{\partial}{\partial t}\right) \frac{d \bar{U}_{x}}{d z}\right|_{z=0} \\
\left.\bar{U}_{x}\right|_{z=-H} & =0,
\end{aligned}
$$

where $\bar{U}_{x}$, the mean displacement in the wall, is

$$
\begin{aligned}
& \bar{V}_{x}=e^{i \Omega t}\left[C_{1} e^{a \sqrt{S t} z}+D_{1} e^{-a \sqrt{S t} z}\right] \\
& \bar{U}_{x}=e^{i \Omega t}\left[E_{1} \sin (\sqrt{S t \Omega} z)+F_{1} \cos (\sqrt{S t \Omega} z)\right],
\end{aligned}
$$


where $a=(1+i) / \sqrt{2}$ and $S t=\frac{\operatorname{Re} \Omega}{\Lambda}$. The constants $C_{1}, D_{1}, E_{1}, F_{1}$, satisfying the four boundary conditions (14) are easily evaluated. The base state is characterised by an oscillatory fluid velocity as well as an oscillatory gel displacement field.

\subsection{Stability analysis}

The time dependent Orr-Sommerfeld equation for the gel is given by

$$
\Sigma_{g} \frac{\partial^{2}}{\partial t^{2}}\left(\partial_{z}^{2}-k^{2}\right) \tilde{u}_{z}=\left(\partial_{z}^{2}-k^{2}\right)^{2} \tilde{u}_{z} .
$$

The fourth order differential operator for the gel displacement $\tilde{u}_{z}$ is factorized, and the resulting equations are

$$
\begin{aligned}
\left(\partial_{z}^{2}-k^{2}\right) \tilde{u}_{z} & =g \\
\frac{\operatorname{Re}}{\Lambda} \frac{\partial}{\partial t}\left(\partial_{z}^{2}-k^{2}\right) b & =\left(\partial_{z}^{2}-k^{2}\right) f .
\end{aligned}
$$

The gel velocity $b=\dot{u}$, gel displacement and the new variable $g$ are expanded in Chebyshev polynomials as

$$
\begin{aligned}
\tilde{u}_{z} & =\sum_{n=0}^{N+2} u_{n} T_{n} \\
g & =\sum_{n=0}^{N+2} g_{n} T_{n} \\
b & =\sum_{n=0}^{N+2} b_{n} T_{n} .
\end{aligned}
$$

A coordinate transformation of $z=(\bar{z}-1) H / 2$ is affected to transform the domain from $z=0$ and $z=1$ to $\bar{z}=-1$ and $\bar{z}=1$. All the derivatives with respect to $\bar{z}$ are indicated by prime in the following section. With this substitution, Eqs. (17) and (18) become

$$
\begin{gathered}
(2 / H)^{2} \sum_{p=n+2}^{N+2} \frac{p\left(p^{2}-n^{2}\right) u_{p}}{c_{n}}-k^{2} u_{n}=g_{n} \\
\frac{\operatorname{Re}}{\Lambda}\left[(2 / H)^{2} \sum_{p=n+2}^{N+2} \frac{p\left(p^{2}-n^{2}\right) \dot{b}_{p}}{c_{n}}-k^{2} \dot{b}_{n}\right]=(2 / H)^{2} \sum_{p=n+2}^{N+2} \frac{p\left(p^{2}-n^{2}\right) g_{p}}{c_{n}}-k^{2} g_{n} \\
b_{n}=\dot{u}_{n} .
\end{gathered}
$$

It is necessary to factorize the gel pressure involving the third derivative of the normal gel displacement to get rid of the spurious eigenvalues (Thaokar \& Kumaran 2004). 


\subsection{Low Reynolds number oscillatory flow over gels}

First, the limit $R e=0$ and $R e_{o}=0$ is considered, where the scaled wave number and frequency are $O(1)$, and $S t=\Sigma_{g} \Omega=0$. In this limit, the mean velocity is

$$
\bar{V}_{x}=\Lambda z+A \operatorname{Real}\left[e^{i \Omega t} \frac{H \Omega-i z+\eta_{g} \Omega z}{H \Omega-i+H \Omega}\right]
$$

and the time dependent mean tangential displacement is

$$
\bar{U}_{x}=-i \text { AReal }\left[e^{i \Omega t} \frac{H+z}{H \Omega-i+\eta_{g} \Omega}\right] .
$$

The governing equations in the zero Reynolds number limit for the perturbations to the velocity and displacement fields are

$$
\begin{aligned}
& \left(\partial_{z}^{2}-k^{2}\right)^{2} \tilde{v}_{z}=0 \\
& \left(\partial_{z}^{2}-k^{2}\right)^{2} \tilde{u}_{z}=0
\end{aligned}
$$

while the boundary conditions are

$$
\begin{aligned}
\tilde{v}_{z} & =\frac{\partial \tilde{u}_{z}}{\partial t}+\bar{V}_{x} i k \tilde{u}_{z} \\
\tilde{v}_{z}^{\prime}-i k\left(\frac{\partial \bar{V}_{x}}{\partial z}-\frac{\partial}{\partial z}\left(\frac{\partial \bar{U}_{x}}{\partial t}\right)\right) \tilde{u}_{z} & =\frac{\partial \tilde{u}_{z}^{\prime}}{\partial t}+\bar{V}_{x} i k \tilde{u}_{z}^{\prime} \\
\left(\tilde{v}_{z}^{\prime \prime}+k^{2} \tilde{v}_{z}\right) & =\left(1+\eta_{g} \frac{\partial}{\partial t}\right)\left(\tilde{u}_{z}^{\prime \prime}+k^{2} \tilde{u}_{z}\right) \\
\left(-\tilde{v}_{z}^{\prime \prime \prime}+3 k^{2} \tilde{v}_{z}^{\prime}\right) & =\left(1+\eta_{g} \frac{\partial}{\partial t}\right)\left(-\tilde{u}_{z}^{\prime \prime \prime}+3 k^{2} \tilde{u}_{z}^{\prime}\right)+\gamma k^{4} \tilde{u}_{z} .
\end{aligned}
$$

The last term in the normal stress balance is due to the effect of surface tension, where $\gamma=$ $\gamma^{*} /(E R)$ is the scaled surface tension. The governing equations (27) and (28) admit the solutions

$$
\begin{aligned}
\tilde{v}_{z}= & c_{1}(t)\left[e^{-k z}-(1+2 k-2 k z) e^{k z-2 k}\right] \\
& +c_{2}(t)\left[z e^{-k z}+(2 k z-2 k-z) e^{k z-2 k}\right], \\
\tilde{u}_{z}= & d_{1}(t)\left[z e^{k z}+(2 k-1) e^{k z} /(2 k)+e^{-(k z+2 k H)} /(2 k)\right] \\
& +d_{2}(t)\left[z e^{-k z}+(2 k H+1) e^{-k z} /(2 k)-e^{k z+2 k H} /(2 k)\right] .
\end{aligned}
$$

The functions $c_{2}(t)$ and $d_{2}(t)$ are evaluated in terms of $c_{1}(t)$ and $d_{1}(t)$ from the boundary conditions (31) and (32), and substituted into Eqs. (29) and (30), to get the dynamical equations which can be written as

$$
\dot{\mathbf{x}}=\mathbf{A x}
$$

where vector $\mathbf{x}=\left(c_{1}(t), d_{1}(t)\right)$ and $\mathbf{A}$ is a matrix of periodic coefficients which are functions of $\cos (\Omega t)$ and $\sin (\Omega t)$. The system can be easily solved to get the Floquet exponents using the Chebyshev Tau method.

3.2a Oscillatory instability: For a purely oscillatory flow, the low Reynolds number asymptotic analysis for this system indicates that the system indeed becomes unstable when the top plate velocity amplitude exceeds a critical value. Figure 1 , which shows the transition amplitude as a 


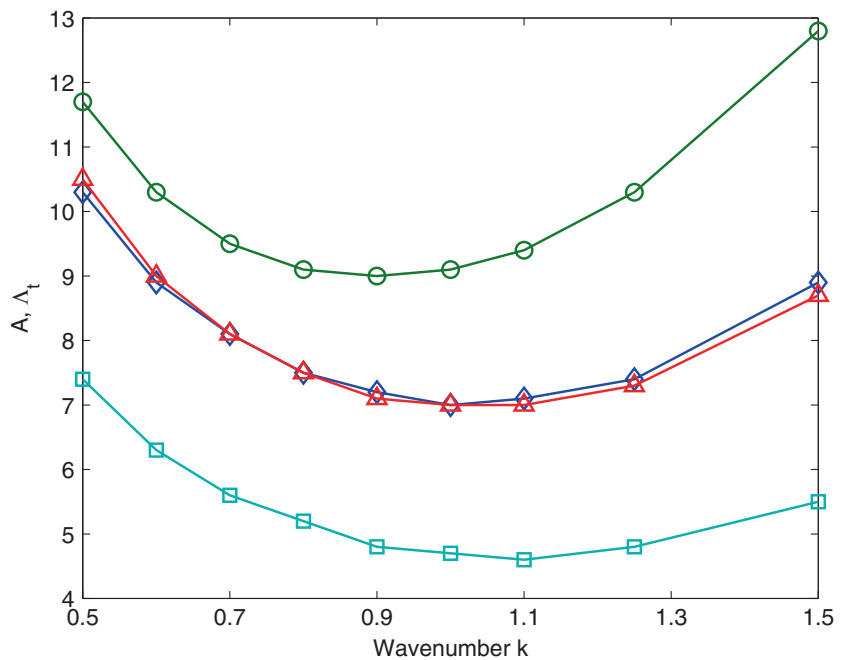

Figure 1. Critical amplitude for low Reynolds number unstable modes for flow over a gel. $\diamond: \Omega=$ 1.0, $\bigcirc: \Omega=5.0, \triangle: \Omega=0.5, \square:$ Steady mean flow, $\Sigma_{g}=0.00025, H=1, \gamma=0.0, \eta_{g}=0$.

function of the wavenumber $k$, indicates that the critical wavenumber is not significantly altered by the oscillations. However the value of critical amplitude initially increases with the increasing frequency, and then decreases at very high frequencies. This figure shows that the critical value of the oscillation amplitude is higher than the critical strain rate required for the non-oscillatory case. The neutral stability curves at the critical wavenumber $k_{c}$ for the purely oscillatory flows for three different gel thicknesses are shown in figure 2 . For a steady flow, the transition velocity is a decreasing function of the gel thickness. However, for an oscillatory flow, it is observed that the transition velocity is a decreasing function of the gel thickness at low frequencies, but

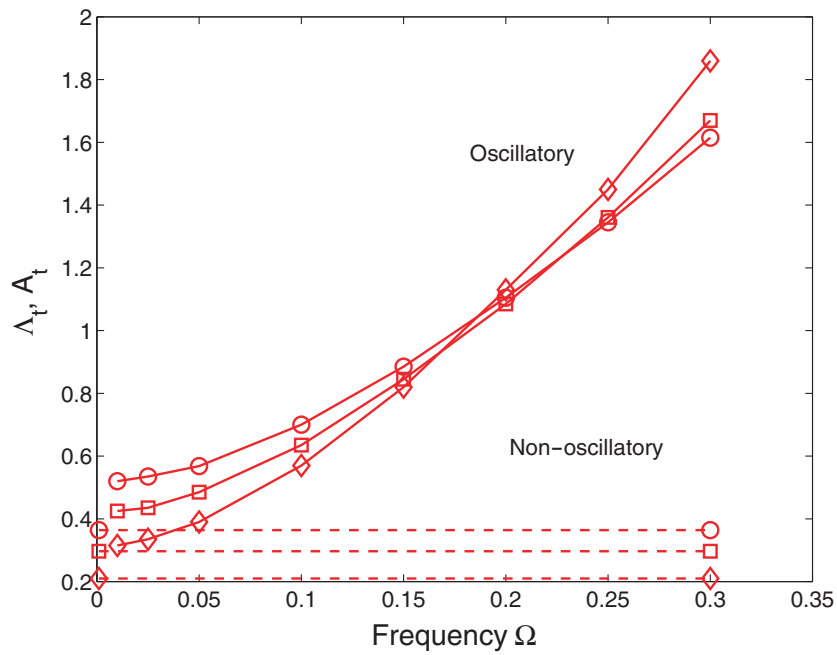

Figure 2. Neutral curves for the steady and oscillatory base flows for $\gamma=0 ; \diamond: \mathrm{H}=15\left(k_{c}=0.1\right), \square: \mathrm{H}$ $=11\left(k_{c}=0.15\right), \bigcirc: \mathrm{H}=9\left(k_{c}=0.2\right), \eta_{g}=0$. 

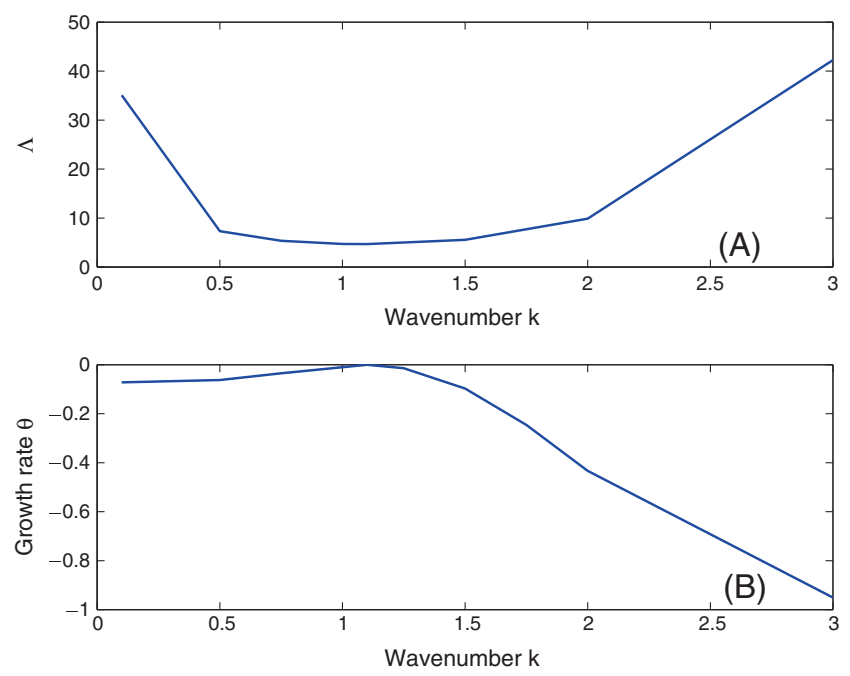

Figure 3. Neutral curve and growth rate for the low Reynolds number unstable modes for flow over a gel for a steady base flow for $\Sigma_{g}=0.025, H=1, \gamma=2.0, \eta_{g}=0$. (a) Neutral stability curve and (b) Growth rate $s$ vs $\left.k, \Lambda_{t}=4.676\right)$.

becomes an increasing function of gel thickness as frequency is increased. The low Reynolds number results are compared with experimental observations in the following section 4.

We now consider the stability of an oscillatory velocity superimposed on a steady velocity of the top plate at two different values of the non-dimensional parameter $\Sigma_{g}$, which is a function only of the physical properties of the system. Our analysis shows that oscillations have a qualitatively different effect on the stability in the low and intermediate Reynolds number regimes. The system is known to exhibit a low Reynolds number instability for a steady base flow, and the critical wave number is comparable to the inverse of the fluid thickness for a fixed value of the parameter $\Sigma_{g}$. The transition Reynolds number scales as $\operatorname{Re} \sim \Sigma_{g}$ in the low Reynolds number regime. We consider three values of the parameter $\Sigma_{g}=0.025,1.0$ and 10.0, corresponding to transition Reynolds number $0.11,4.738$ and 37.2 respectively, in the low and intermediate Reynolds number ranges.

Figure 3(a) shows the transition strain rate as a function of wave number for $\Sigma_{g}=0.025$, which corresponds to transition Reynolds number $R e_{t}=0.11$ for a steady flow, and figure 3(b) shows the growth rate as a function of the wave number for the critical $\Lambda_{c}=4.676$ at which the $\Lambda-k$ shows a minimum. The effect of plate oscillation on the growth rate is examined in figure 4 when the strain rate in the steady flow is 4.676 and the wave number is $k_{c}=1.1$. This figure shows that plate oscillations increase the growth rate, and have a destabilizing effect. The growth rate is also found to increase with an increase in the amplitude of oscillations. The dependence on the frequency is more complex, since the growth rate first increases and then decreases with frequency. The above results indicate that in the low Reynolds number regime, the critical strain rate is predominantly reduced in the presence of plate oscillation, and table 1 shows the dependence of the critical strain rate on the amplitude of the wall oscillation.

Figure 5(a) shows the variation of transition strain rate with wave number for a steady flow for a higher value of parameter $\Sigma_{g}=1.0$, and figure $5(\mathrm{~b})$ shows the growth rate as a function of wave number at the critical mean strain rate $\Lambda_{c}=4.676$. The transition Reynolds number $R e_{t}=4.738$ for this case. The effect of plate oscillations on the growth rate at the critical strain 


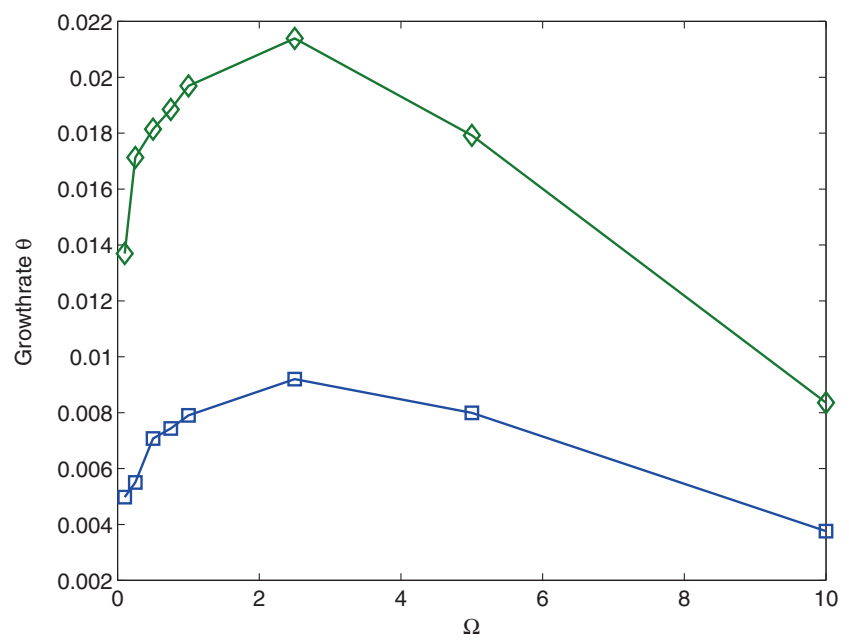

Figure 4. Effect of oscillation frequency on the growth rate of the instability of the steady flow past a gel at low Reynolds number; $\left(\square: A=0.5 \Lambda, \diamond: A=0.75 \Lambda, \Sigma_{g}=0.025 H=1 \Lambda_{t}=4.676, \mathrm{k}=\right.$ $\left.1.1, \gamma=2.0, \eta_{g}=0\right)$.

rate and wave number $k_{c}=1.1$ are shown in figure 6 . This figure shows that the plate oscillations have a complicated effect on the growth rate in this parameter regime. Wall oscillations are found to have a stabilising effect at high frequencies and destabilizing at lower frequencies. The system shows a maximum in the growth rate at an intermediate frequency. The growth rate is also found to increase with an increase in the amplitude of oscillations.

The dependence of the transition velocity on the frequency of oscillations is more complicated at high Reynolds number, due to the non-monotonic dependence of the mean strain rate at the interface on the frequency of oscillations. Figure 7 shows the variation of growth rate of a neutrally stable non-oscillatory mode with frequency for two different wall amplitudes. The plot shows a strong destabilizing effect at a oscillation frequency of $\Omega=1.5$. This can be understood from the mean velocity and the mean velocity gradient profiles shown in figure 8 , which show that the mean strain rate at the interface also has a maximum with respect to frequency at $\Omega=1.5$. Other calculations also show that the positions of the peaks in the $s-\Omega$ curve coincide with the positions of the peaks in the $A^{\prime}-\Omega$ curves.

Table 1. Effect of frequency on critical strain rate, $k_{c}=1.1$, $\Sigma_{g}=0.025, \mathrm{H}=1.0 \gamma=2.0, \eta_{g}=0$.

\begin{tabular}{lcc}
\hline Frequency $(\Omega)$ & $A=0.5 \Lambda$ & $A=0.75 \Lambda$ \\
\hline Non-oscillatory & 4.677 & \\
0.1 & 4.625 & 4.52 \\
0.5 & 4.59 & 4.48 \\
1.0 & 4.59 & 4.47 \\
2.5 & 4.58 & 4.45 \\
5.0 & 4.58 & 4.46 \\
10.0 & 4.62 & 4.56 \\
\hline
\end{tabular}



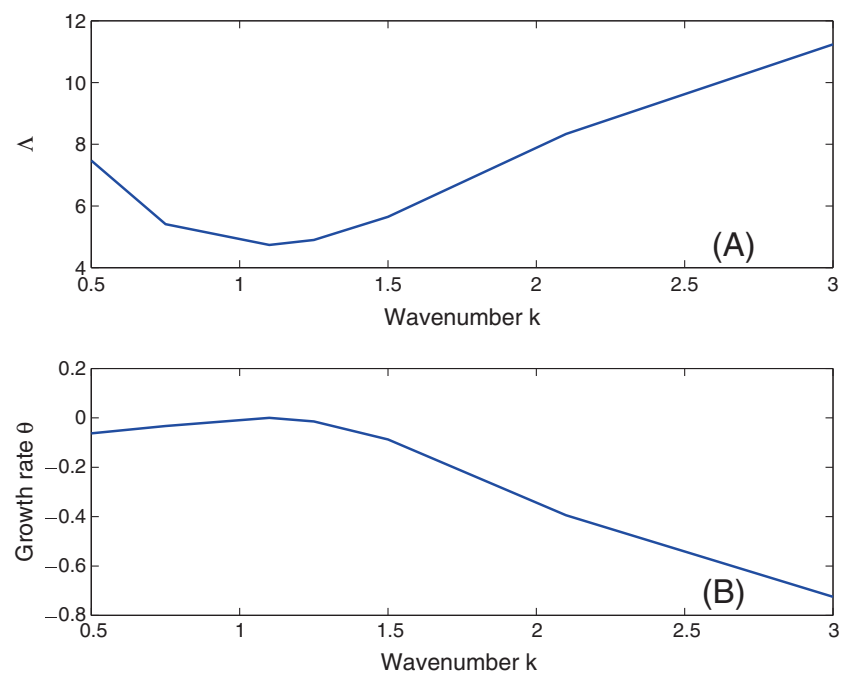

Figure 5. Neutral stability curve and growth rate for the steady flow past a gel for $\Sigma_{g}=1.0, H=1, \eta_{g}=$ 0 . (a) Neutral curve and (b) Growth rate at $\Lambda_{t}=4.738$.

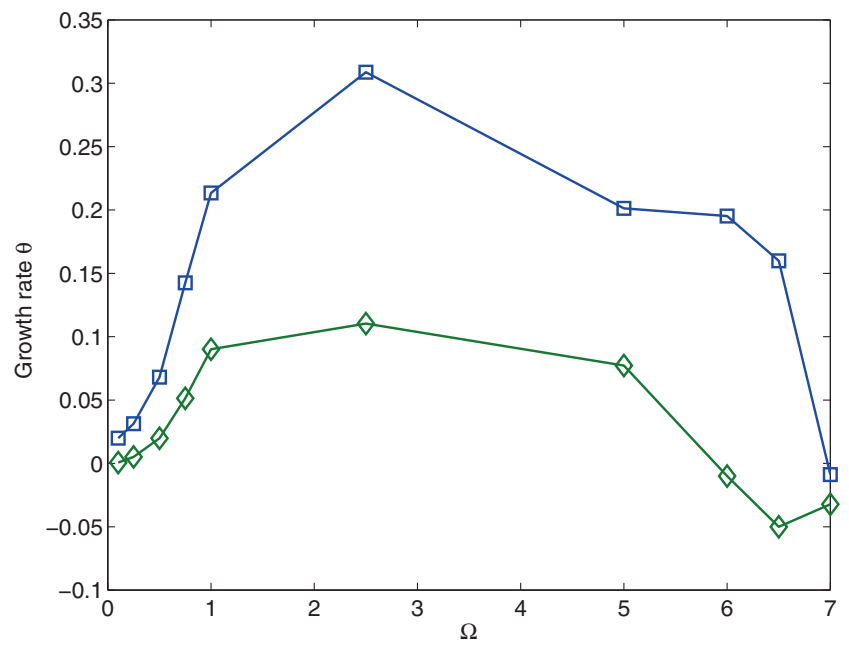

Figure 6. Effect of oscillation frequency on the growth rate of the steady flow past a gel, $\square: A=0.7 \Lambda$, $\diamond: A=0.5 \Lambda, \Sigma_{g}=1.0, H=1, \Lambda=4.738, k=1.1, \eta_{g}=0$.

\section{Experimental studies}

\subsection{Preparation of gels}

Polyacrylamide gels were used as the viscoelastic surface for studying the oscillatory flow instability at the fluid-gel interface. These gels were especially chosen because the elastic properties of these gels can be controlled by changing the relative compositions of the monomer and cross-linker. The gels are formed by polymerization of $\mathrm{N}$-Acrylamide monomer, $\mathrm{Bis}\left(N, N^{\prime}\right.$ Methylenebisacrylamide) is used as the cross linker, Ammonium Persulfate (APS) as the initiator 

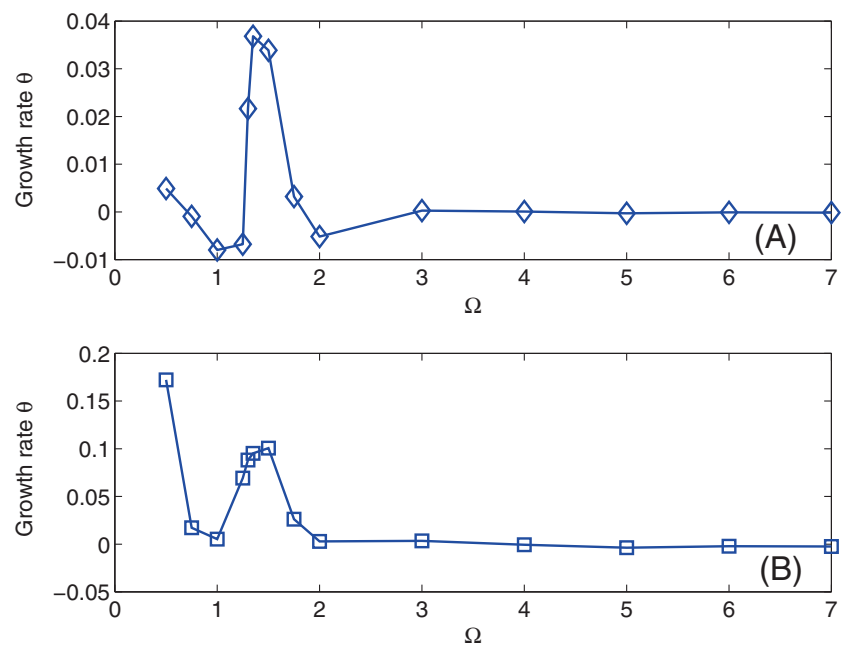

Figure 7. Variation of growth rate with oscillation frequency for two different amplitudes of the oscillatory velocity, (a) $A=0.25 \Lambda$, (b) $A=0.5 \Lambda$, for $\Sigma_{g}=10.0, H=1, \gamma=2.0, \Lambda=3.727846, k=1.75$, $\eta_{g}=0$.

and Tetramethylenediamine (TEMED) as the catalyst. The gelation reaction is a vinyl-divinyl radical polymerization reaction, which results in macroscopic polymer networks, when suitable compositions are used.

$20 \% \mathrm{w} / \mathrm{w}$ solution of monomer was prepared by dissolving 20 gm Acrylamide in $100 \mathrm{ml}$ distilled water. $1 \% \mathrm{w} / \mathrm{w}$ Bis was prepared by dissolving $1 \mathrm{gm}$ Bis in $100 \mathrm{ml}$ distilled water. TEMED solution was prepared by dissolving $0.5 \mathrm{ml}$ in $200 \mathrm{ml}$ distilled water and $1 \%$ catalyst solution was prepared by dissolving $1 \mathrm{gm}$ APS in $100 \mathrm{ml}$ distilled water. The solution mixture was prepared by adding $9 \mathrm{ml}$ Bis, $4 \mathrm{ml}$ APS solution, and $4 \mathrm{ml}$ TEMED solution. The monomer quantity was varied between 5 and $8 \mathrm{ml}$ and the solution was diluted to $30 \mathrm{ml}$ with distilled water. This gave gels with Acrylamide percentage between 4 and $8 \%$ which had the desired properties. The solution was then rapidly poured into a specially prepared mould of size $5 \mathrm{~cm} \times 5 \mathrm{~cm} \times 4.5$ $\mathrm{mm}$. The gelation time varied between 10-30 mins.

The oscillatory stability studies as well as the characterization of the gel material were carried out on a Rheolyst AR 1000N Rheometer. The Rheometer has two important parts, the bottom Peltier plate and the geometry (figure 9). The Peltier plate is used to control the temperature of the sample using the Peltier effect to within $\pm 0.1^{\circ} \mathrm{C}$. The geometry, which consists of a plate of diameter $4 \mathrm{~cm}$, is attached to the head of the Rheometer using a draw rod. There is a normal force transducer below the Peltier plate to measure the normal stress. The measurement procedure is as follows. A film of fluid between $100 \mu \mathrm{m}$ and $1 \mathrm{~mm}$ is placed between the top and bottom plates, and held in place by surface tension. The top plate is rotated at a constant angular velocity, and the torque on the top plate is measured. From this, the stress and strain rate at the outer edge of the top plate are calculated assuming the flow is laminar, and the viscosity (and shear moduli in oscillatory experiments) are determined from the ratio of the stress and strain rate. In the steady and oscillatory experiments on the flow past a gel, the gel is placed on the bottom Peltier plate, and the fluid is placed on the gel. The top plate is lowered to obtain a fluid film of the desired thickness, and the viscosity measurements are conducted. The stress is increased at a constant rate, and the viscosity is calculated by the rheology measurement software as indicated above. 

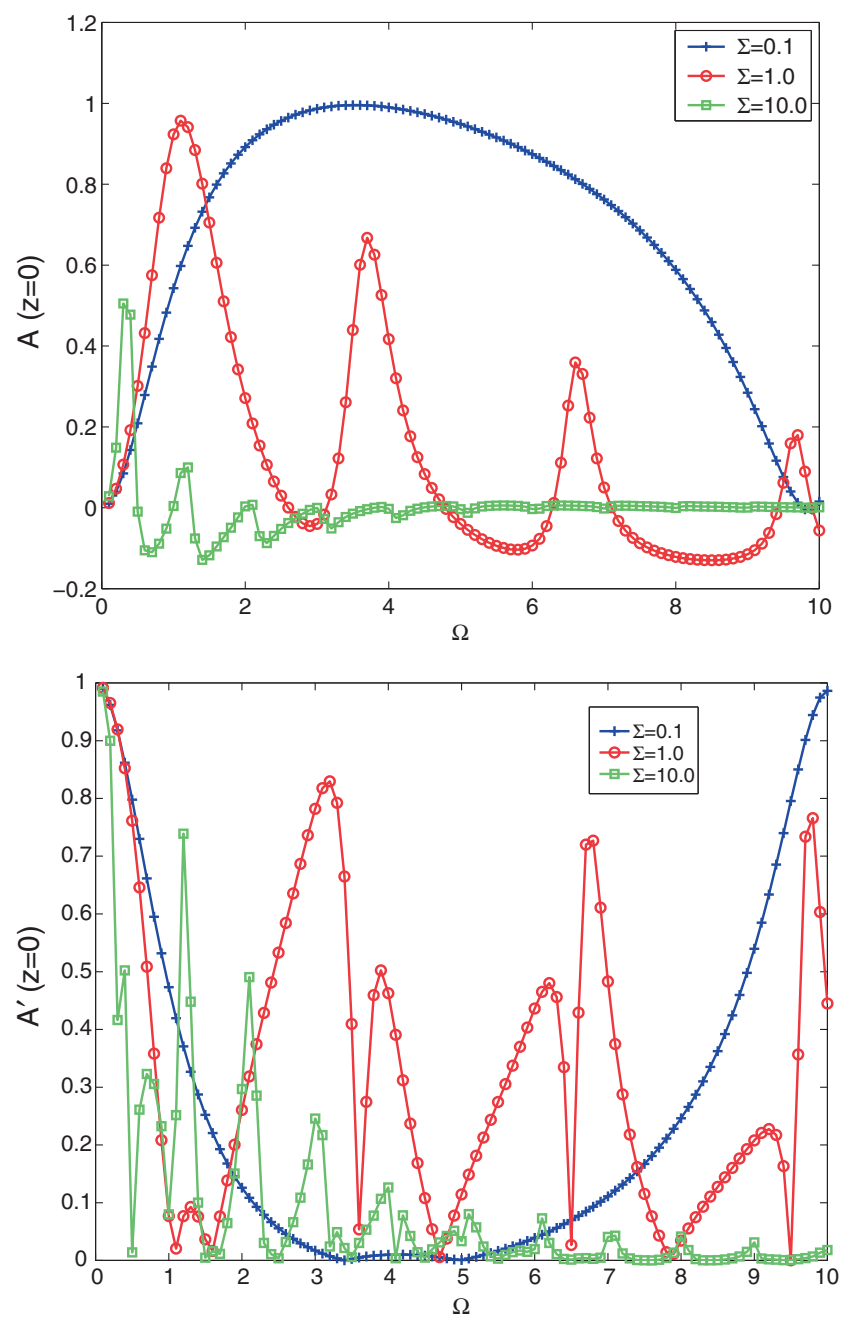

Figure 8. The amplitude of the velocity fluctuations $A$, and the derivative of $A$ with respect to the $z$ coordinate $A^{\prime}$, as a function of the oscillation frequency $\Omega$ for different values of $\Sigma_{b}$. $\left(+: \Sigma_{g}=0.1, \bigcirc\right.$ : $\left.\Sigma_{g}=1.0, \square: \Sigma_{g}=10.0, H=1, \gamma=2.0, \eta_{g}=0\right)$.

If the flow is laminar, the viscosity is a constant and is equal to the value obtained for the flow between two rigid plates. When a transition to a more complicated flow pattern takes place, the reported viscosity (which is the ratio of the apparent stress and strain rate calculated assuming the flow is laminar) is higher than that which would be measured for a laminar flow, indicating that a transition has taken place.

The Polyacrylamide gels were first characterised as follows. The geometry was first lowered till it touches the Peltier plate, and the zero gap adjustment was calibrated. The geometry was then raised, and the gel sample was placed on the Peltier plate. The geometry was then lowered till it makes contact with the top of the gel surface, and contact was detected using the normal force measurement, providing the height of the gel $H$. The shear modulus $G^{\prime}$ was then measured 


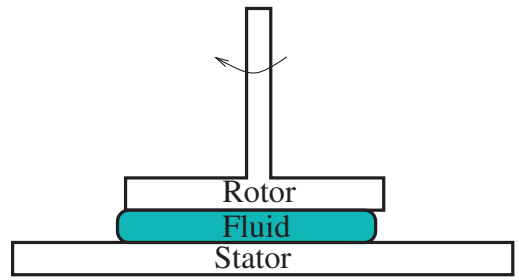

(A)

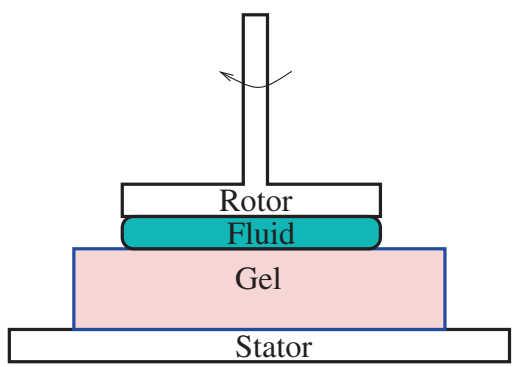

(B)

Figure 9. Schematics of the Rheometer: (a) unmodified (b) modified.

as a function of frequency using a stress amplitude of $3 \mathrm{~N}$, and the results of one such measurement are shown in figure 10. For the gels studied in this work, the $G^{\prime}$ value was found to be independent of frequency in the frequency range $0.1 \mathrm{~Hz}$ to $10 \mathrm{~Hz}$. The loss modulus was more difficult to measure due to limitations in the instrument, and this was determined indirectly as explained below. The geometry was raised at the end of the experiment, and the fluid (Silicone oil of viscosity $1 \mathrm{~Pa}$ ) was placed on the gel surface. The geometry was lowered to obtain a preset fluid thickness $R$, which was varied in the experiments between $R=300 \mu \mathrm{m}$ and $500 \mu \mathrm{m}$. The instrument was then put into oscillation mode and the top plate was given a stress amplitude ramp at constant frequency $\tau_{o}=\tau_{t} \cos (\Omega t)$. The viscosity was then monitored, and the presence of an instability was inferred as discussed above, and a typical viscosity-stress curve showing the onset of instability is shown in figure 11.

The oscillatory experiment was repeated at different frequencies for the same sample, and care was taken to ensure that the experiment was stopped before there is significant damage to the surface of the gel due to the instability. After the oscillation experiments are over, the stability of a steady flow was measured using a steady stress ramp, where the stress is increased at a constant

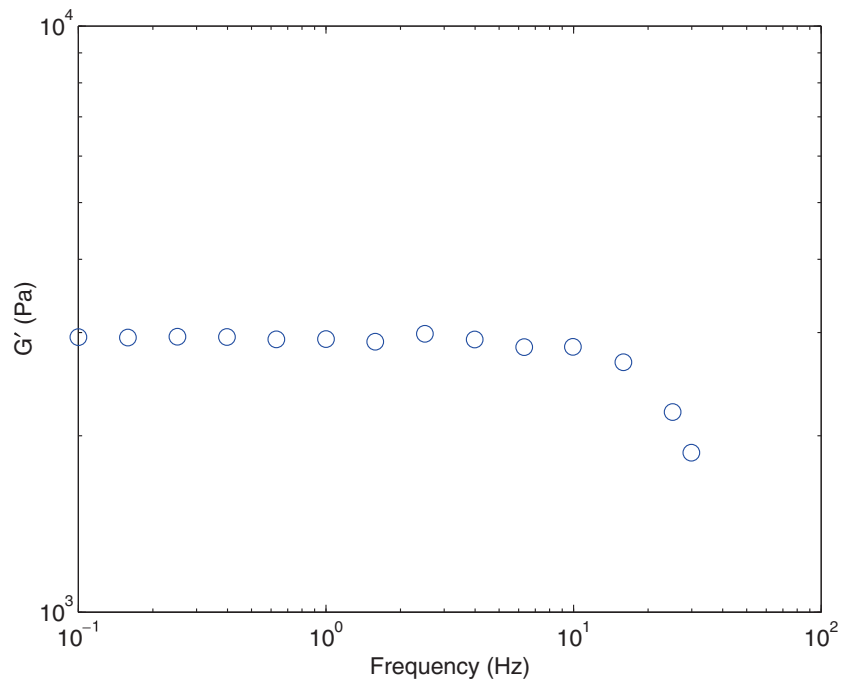

Figure 10. Variation of storage modulus with frequency $\mathrm{H}=4680 \mu \mathrm{m}$. 


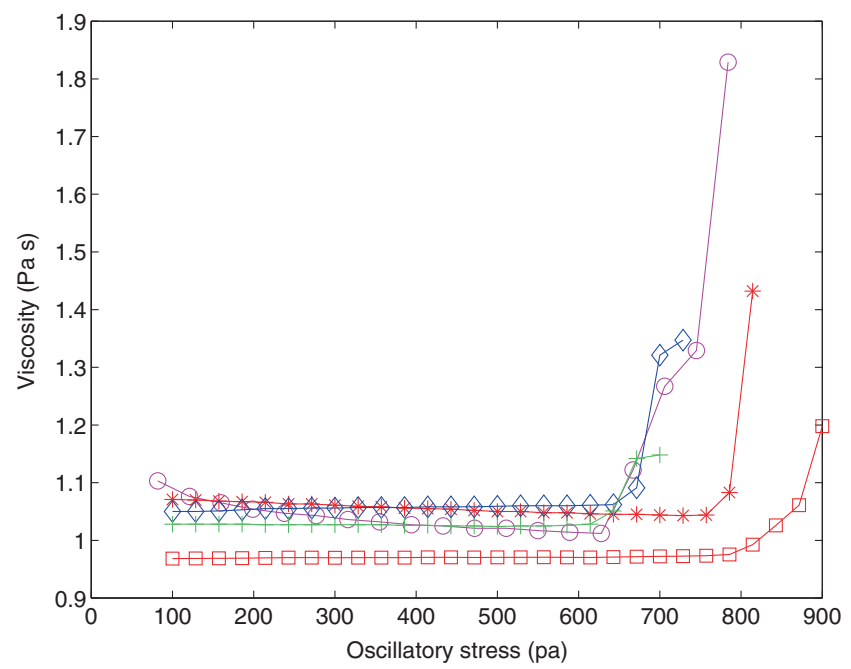

Figure 11. Viscosity as a function of stress amplitude for $H=4709 \mu \mathrm{m}, G=2205 \mathrm{~Pa}, \eta=1.0 \mathrm{~Pa} \mathrm{~s}$, $R=300 \mu \mathrm{m} ; \bigcirc:$ Steady flow, $*: \Omega=0.0028, \diamond: \Omega=0.0057,+: \Omega=0.0142, \square: \Omega=0.0214$.

rate and the viscosity. The observation of the instability of the mean flow at the stress predicted by theory was used to indicate that the surface of the gel is not damaged by the flow.

Since the experiments are conducted under constant stress conditions, it is necessary to modify the Floquet analysis to calculate the critical stress required for the onset of instability, rather than the critical strain rate which was calculated in the previous section. This calculation is easily carried out, and is not explained in detail here. It is important to note, however, that the results show a strong dependence of the transition stress on the gel viscosity. For example, the effect of gel viscosity on the transition stress, shown in figure 12, indicates that there is a significant

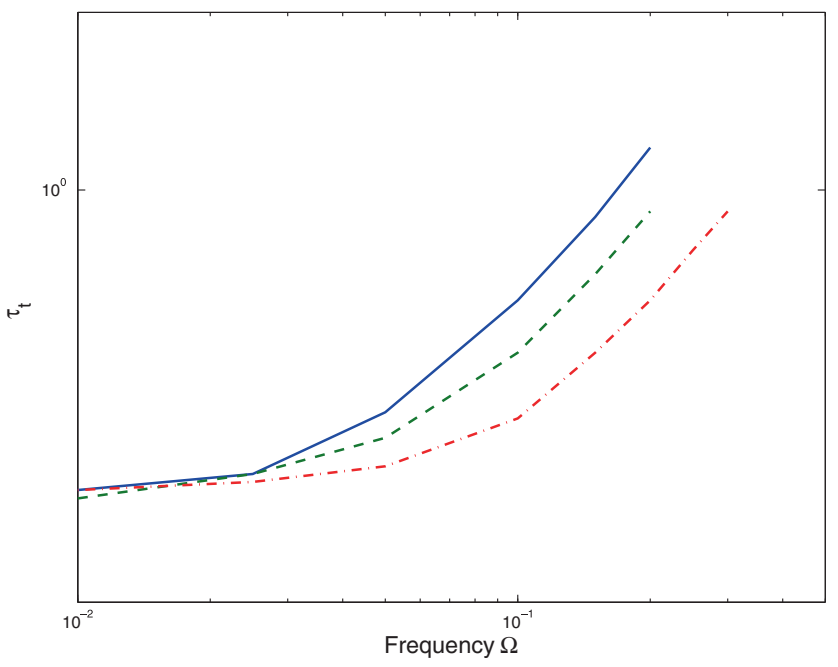

Figure 12. Critical stress amplitude vs frequency for $\mathrm{H}=15$ for different gel viscosities, $\left(-: \eta_{g}=16.0\right.$, $-: \eta_{g}=10, \cdots \cdot \eta_{g}=5.0$ ). 
difference in the critical stress for the oscillatory instability when the viscosity ratio is increased from 5 to 16 , though there is less variation in the critical stress for a steady flow in the zero frequency limit. The gel viscosity could not be determined from experiments, since the loss modulus of the soft gels used in the experiments could not be determined due to limitations in the oscillatory measurements. In order to compare the theory with experiments, the viscosity ratio has been set equal to 16 , since this value is consistent with the theoretical predictions for the critical stress for a steady flow. However, the sensitive dependence of the critical stress on the viscosity ratio should be kept in mind while interpreting the results.

Figures 13 and 14 show the experimental results for two different nondimensional $H$ values, $H=15$ and $H=9$, which in the present case correspond to fluid thicknesses of $300 \mu \mathrm{m}$ and $500 \mu \mathrm{m}$ respectively. The different data sets in the plots correspond to different gel thicknesses and shear moduli. The results are compared with the theoretical results obtained using the procedures in section 3. These figures show the theoretical predictions for the critical stress for a steady flow, as well as the frequency dependence of the critical stress for an oscillatory flow. The experimental results show significant scatter, but are consistent with a transition from the critical stress for a steady flow to that for an oscillatory flow as the frequency increases. This is expected for the following reason. At low frequencies, the growth rates corresponding to the steady flow instability are much larger than the time scales of the oscillation, and the mean flow at any instant of time can be considered to be a steady Couette flow driven by the stress exerted by the top plate. In this case, it is expected that the onset of instability occurs when the maximum stress at the top plate exceeds the critical stress for a steady flow required for the onset of instability. As the

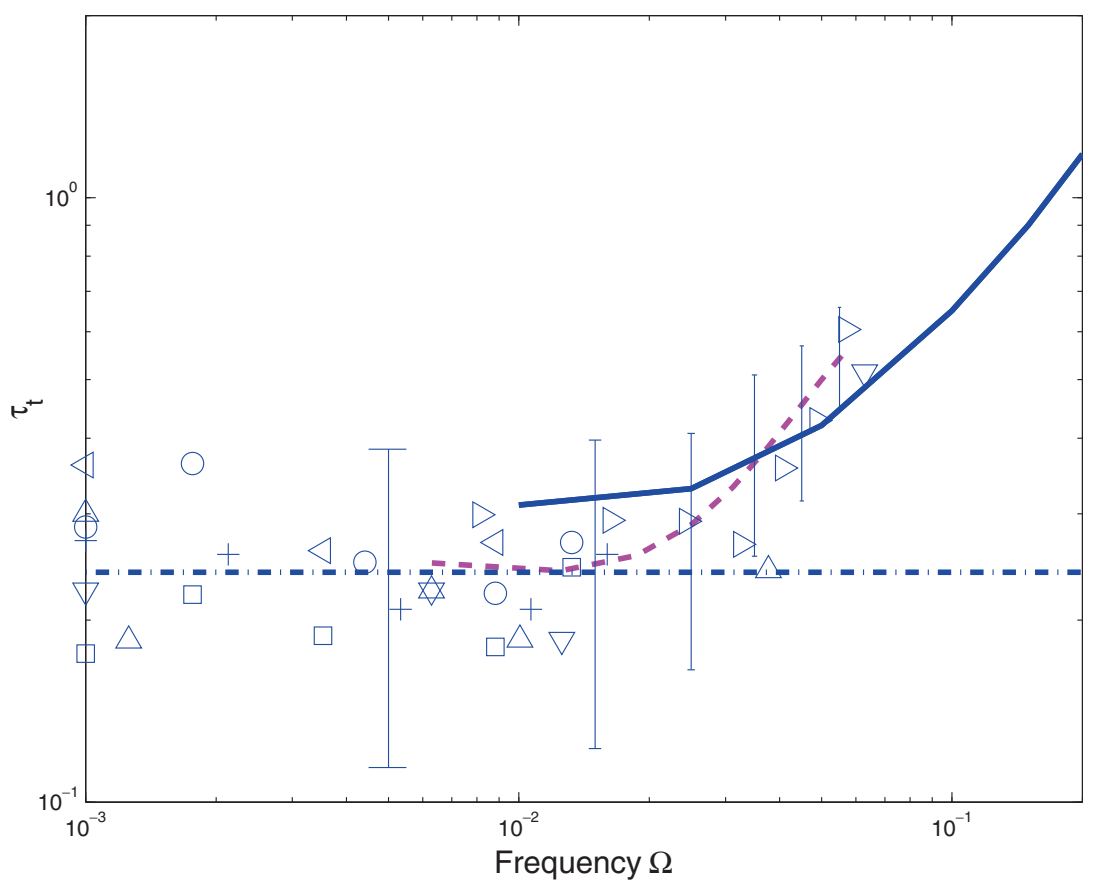

Figure 13. Critical stress amplitude vs frequency for $\mathrm{H}=15$; - : theoretical prediction steady base flow with $\eta_{g}=16,-\cdots$ : theoretical prediction steady base flow with $\eta_{g}=16$, --: best fit of experimental data, $\square: H=4614, \mathrm{G}^{\prime}=3500, \bigcirc: \mathrm{H}=4600, \mathrm{G}^{\prime}=3560,+: \mathrm{H}=4600, \mathrm{G}^{\prime}=2945, \triangle: \mathrm{H}=4400, \mathrm{G}^{\prime}=$ $500, \nabla: H=4600, G^{\prime}=600, \triangleright: H=4721, G^{\prime}=700, \triangleleft: H=4702, G^{\prime}=1000, \diamond: H=4525, G^{\prime}=1250$. 


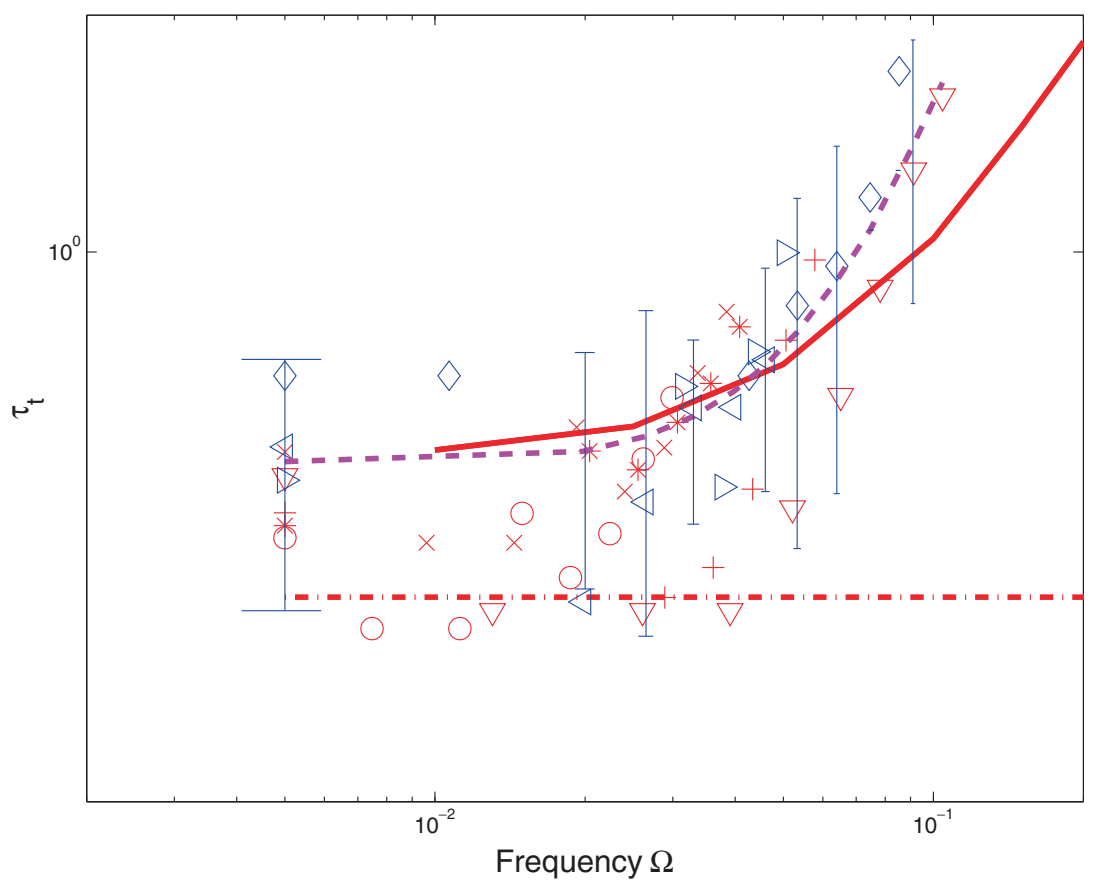

Figure 14. Critical stress amplitude vs frequency for $\mathrm{H}=9$; —: theoretical prediction oscillatory base flow with $\eta_{g}=16, \cdots \cdot-$ : theoretical prediction steady base flow with $\eta_{g}=16$, --: best fit for experimental results, $*: \mathrm{H}=4728, \mathrm{G}^{\prime}=1230, \nabla: \mathrm{H}=4720, \mathrm{G}^{\prime}=482,+: \mathrm{H}=4637, \mathrm{G}^{\prime}=869, \times: \mathrm{H}=4627, \mathrm{G}^{\prime}=$ 1307, $\bigcirc: \mathrm{H}=4746, \mathrm{G}^{\prime}=1681, \diamond: \mathrm{H}=4701, \mathrm{G}^{\prime}=589, \triangle: \mathrm{H}=4714, \mathrm{G}^{\prime}=3500, \triangleright: \mathrm{H}=4715, \mathrm{G}^{\prime}=$ $1000, \triangleleft: \mathrm{H}=4043, \mathrm{G}^{\prime}=950$ ).

frequency is increased, the growth rate of perturbations is not large compared to the frequency of oscillations, and the growth of fluctuations when the top plate stress is greater than the steady critical stress is offset by the decay of fluctuations when the top plate stress is lower than the steady critical stress. In this case, it is expected that the instability is due to the accumulated growth of perturbations over many cycles, which is studied in the Floquet analysis conducted here.

There is significant scatter in the data for many reasons. The gels used here are soft, with shear modulus between 400 and $3000 \mathrm{~Pa}$. In this case, it is difficult to get an accurate estimate of the gel thickness, because the gel gets significantly compressed when the zero gap adjustment is made. Since the gel is about 10 times thicker than the fluid film, an error of $1 \%$ in the gel thickness could result in a variation of $10 \%$ in the fluid thickness, thereby resulting in an error in the theoretical estimate of the critical strain rate. In addition, as noted before, there is a sensitive dependence of the critical stress on the viscosity ratio. Despite these uncertainties, it is clearly seen that there is a transition from the critical stress for a steady flow to the critical stress for the oscillatory flow as the frequency is increased, and the predicted increase in the critical stress with frequency is clearly observed in the experiment. When the critical stress is averaged over all the experimental runs, there is agreement between the theoretical and experimental results with no fitting parameters, apart from the gel viscosity which was fitted to obtain agreement for the steady flow. This provides experimental confirmation of the theoretical predictions of the Floquet analysis. 


\section{Electrohydrodynamic instability in flow over viscoelastic gels}

Consider Newtonian fluid of thickness $R^{\alpha}$ placed over a viscoelastic gel of thickness $H R^{\alpha}$ grafted over a rigid wall so that the zero displacement conditions are valid at the rigid wall. The top plate is moved at velocity $V$. The velocity is scaled by $R^{\alpha} G / \mu^{\alpha}$, where $\mu^{\alpha}$ is the viscosity of the fluid and $G^{*}$ is the shear modulus of the viscoelastic gel. We scale here the electric potentials with $\left(R^{\alpha 2} G^{*} / \epsilon_{0}\right)^{1 / 2}$. The system is known to exhibit viscous instability in the presence of flow in the zero Reynolds number limit due to the work done by the fluid at the deformable gel interface (Kumaran et al 1994) when the non-dimensional strain rate $\Lambda=\left(\mu V /\left(R^{\alpha} G^{\alpha}\right)\right)$ exceeds a critical strain rate $\Lambda_{c}$. The instability is a finite wavenumber instability and occurs at $\Lambda \sim O(1)$.
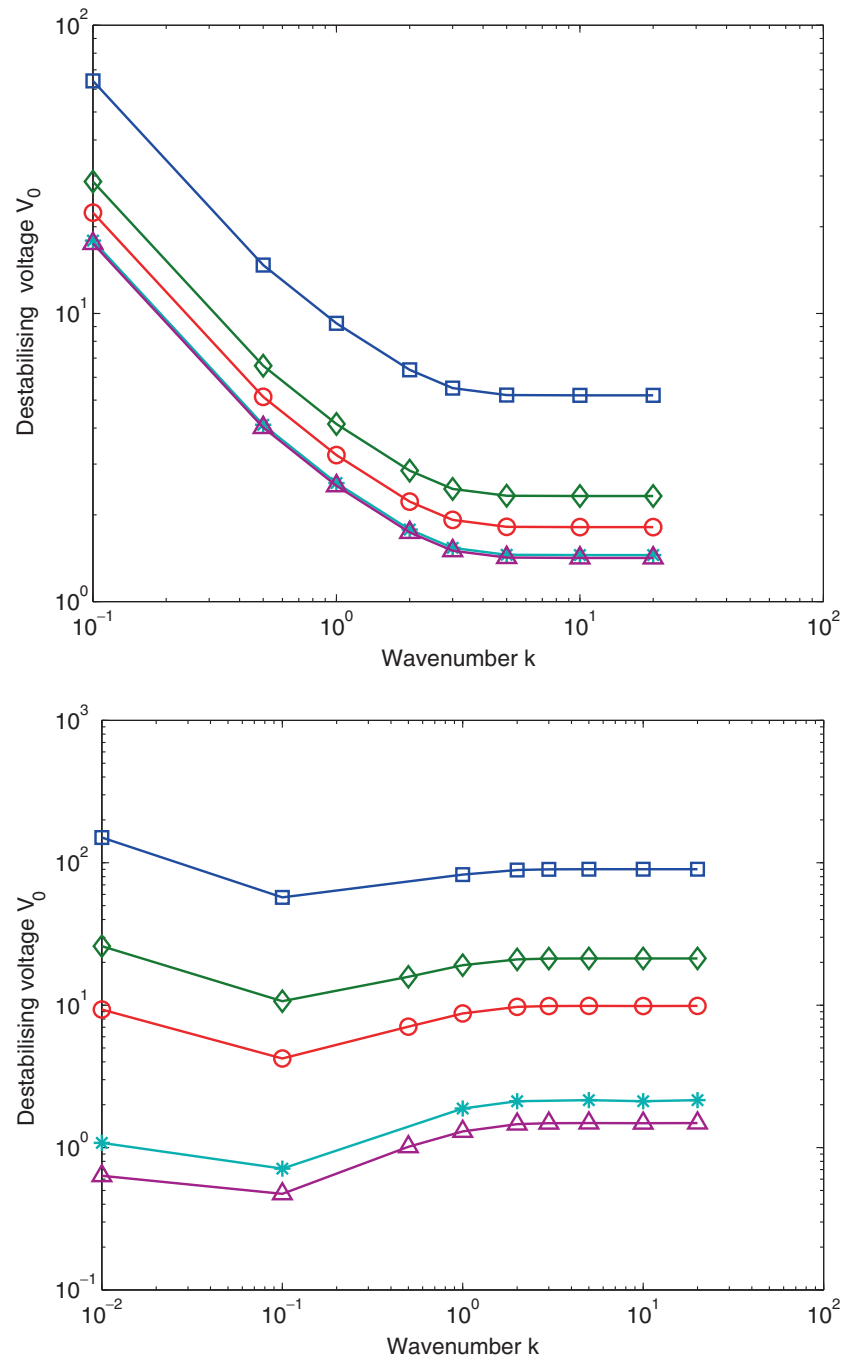

Figure 15. Effect of dielectric constant ratio on the electrohydrodynamic instability in the absence of flow in a fluid-gel system $\left(\epsilon_{B}=1, \gamma=0, \eta_{g}=0, \Lambda=0\right)$, (a) $\mathrm{H}=1$ and (b) $\mathrm{H}=50 ; \square: \epsilon_{R}=0.5, \diamond: \epsilon_{R}=$ $0.2, \bigcirc: \epsilon_{R}=0.1, *: \epsilon_{R}=0.01, \triangle: \epsilon_{R}=0.001$. 
The details of the instability are given in Kumaran et al (1994). The problem formulation and notations used in this work are exactly similar to the work of Kumaran et al (1994) and is not repeated here.

It is also known that an electrohydrodynamic instability exists at the interface of two dielectric fluids when subjected to a critical destabilizing potential (Melcher \& Smith 1968; Saville 1997). The electrohydrodynamic instability is known to be a low wave number instability in the two dielectric fluid case and is expected to exist even for the fluid-gel system. Thus, it is interesting to see the dependence of the electrohydrodynamic instability on the gel parameters as well as to see the modification of the flow instability due to electrohydrodynamic effects, and the modification of the electrohydrodynamic instability due to shear flow. The linear stability analysis indicates that fluid-gel system shows an electrohydrodynamic instability in the absence of fluid flow. Both the gel and the fluid are assumed to be perfect dielectrics, and the instability occurs when the destabilizing Maxwell stresses at the gel-fluid interface exceed the restoring forces which are due to the elastic strain energy in the solid and the viscous forces in the fluid. Figure 15 shows neutral stability curve for the electrohydrodynamic instability for two different gel thicknesses, $H=1$ and $H=50$, and the region above the neutral stability curve is unstable.

In both the cases, the system becomes more unstable as the ratio of dielectric constant is increased. The instability is a finite wavenumber instability due to elastic stresses in the gel, unlike the case of the interface between two fluids which exhibits a low wavenumber instability. There is a qualitative difference in the neutral stability curves for small and large gel thicknesses, as shown in figure 16. At very small gel thickness, the neutral curve monotonically decreases and converges to a constant value at large wavenumbers, while the destabilizing voltage diverges at small values of wavenumbers as $V_{c} \sim k^{-1}$. At higher gel thicknesses, the potential difference on the neutral stability curve has a minimum at a finite wave number, and increases in the low and high wave number limits. This critical wavenumber is dependent on the gel thickness, but independent of the ratio of dielectric constants. The neutral stability curves for the viscous instability in the absence of electric field are shown in figure 17 . The strain rate required to destabilize the system diverges at very low and very high wavenumbers. The system thus shows a critical

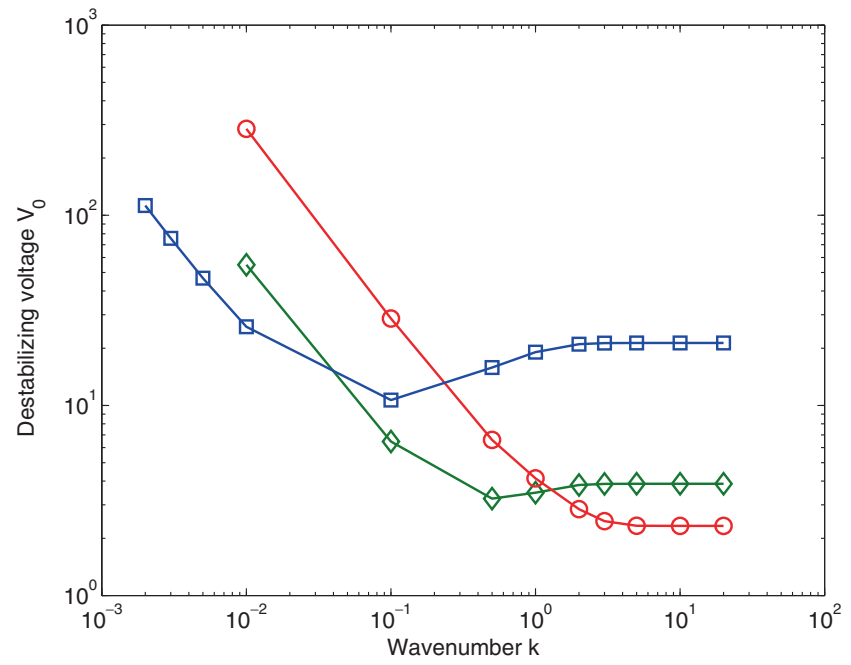

Figure 16. Effect of gel thickness on the electrohydrodynamic instability in the absence of flow in a fluid-gel system $\left(\gamma=0, \eta_{g}=0, \Lambda=0, \epsilon_{A}=1, \epsilon_{R}=5\right) ; \square: \mathrm{H}=50, \diamond: \mathrm{H}=5$ and $\bigcirc: \mathrm{H}=1$. 


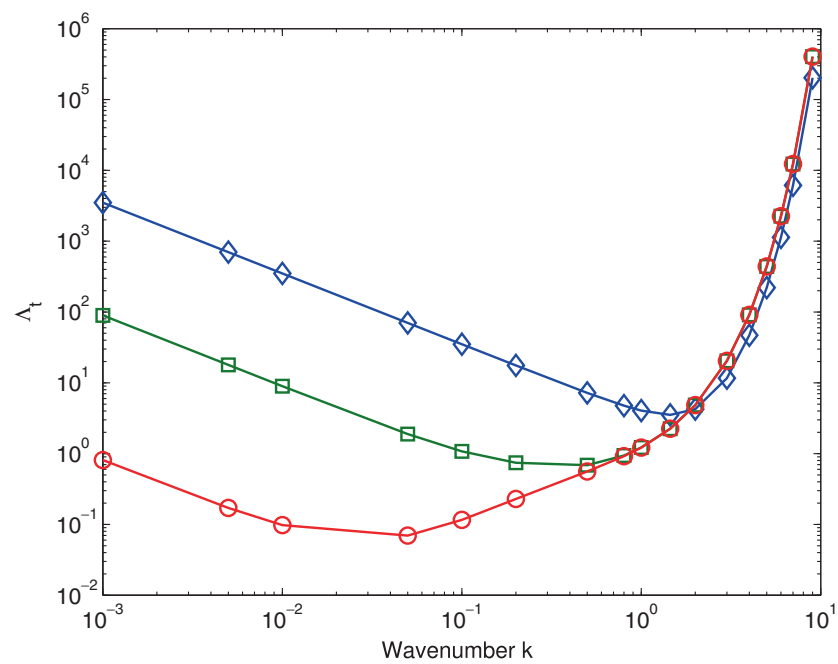

Figure 17. Effect of gel thickness on the viscous instability in the absence of electric field in a fluid-gel $\operatorname{system}\left(\gamma=0, \eta_{g}=0, V=0\right) ; \diamond: \mathrm{H}=1, \square: \mathrm{H}=5$ and $\bigcirc: \mathrm{H}=50$.

wavenumber corresponding to the most unstable mode. The critical wavenumber corresponding to the critical strain rate required to destabilize the system varies as $H^{-1}$. The effect of strain rate and the applied voltage on the electrohydrodynamic and the viscous instability are shown in figures 18 and 19.

The results indicate that the two instabilities have a positive reinforcement effect on each other so that the unstable modes are destabilized by both the viscous instability and the electrohydrodynamic instability simultaneously. Figure 18 shows the effect of fluid flow on the
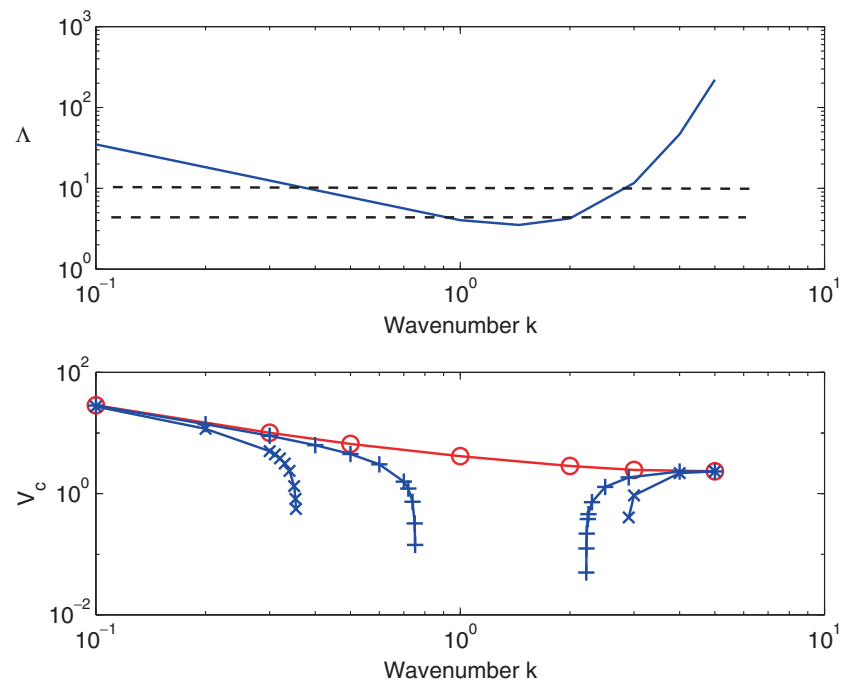

Figure 18. Effect of shear rate on the electrohydrodynamic instability; $\bigcirc: \Lambda=0.1,+: \Lambda=5, \alpha \Lambda=$ $10, H=1, \eta_{g}=0, \epsilon_{A}=1, \epsilon_{R}=2$. 


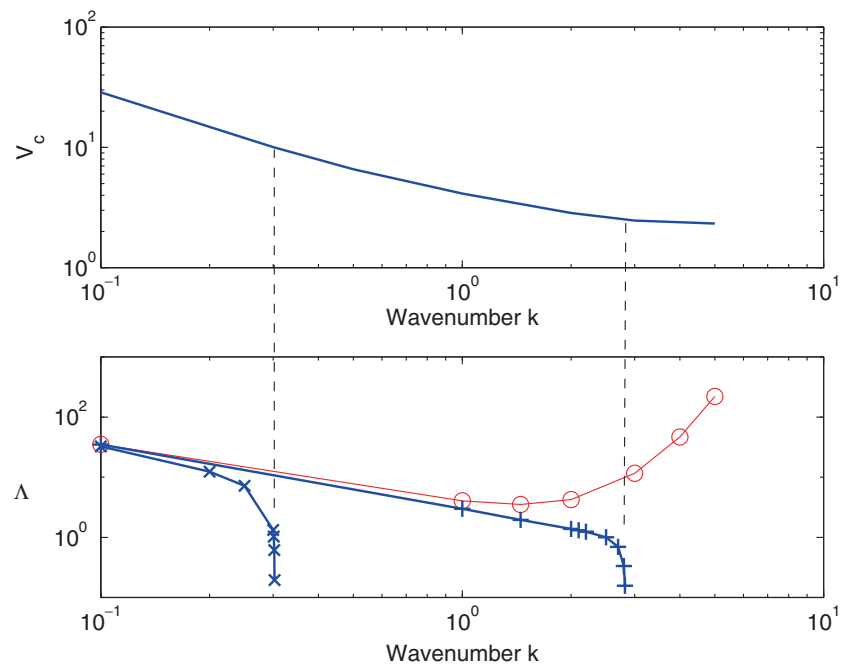

Figure 19. Effect of applied voltage on the viscous instability $\left(\bigcirc: V_{0}=0,+: V_{0}=2.5, \alpha V_{0}=10.0\right.$, $H=1, \eta_{g}=0, \epsilon_{A}=1, \epsilon_{R}=2$ ).

electrohydrodynamic instability. At low strain rates, the instability is weakly affected by the strain rate. However, as the strain rate is increased, there is a range of wave numbers that is unstable. In the $V \rightarrow 0$ limit, this corresponds to the range of wave numbers that is destabilized by the viscous instability, which cannot be stabilised by applying a potential. As the potential is increased, the range of unstable wave numbers also increases. In a similar manner, when a potential is applied across a gel-fluid system which is already subjected to a mean flow, it is found that perturbations with wave number above a maximum value $k_{m}$ become unstable. In the limit $\Lambda \rightarrow 0, k_{m}$ corresponds to the maximum stable wave number in the absence of flow, as shown in figure 19. However, as $\Lambda$ is increased, the value of $k_{m}$ decreases due to the additive effect of the flow instability and the electrical instability.

\section{Conclusions}

The effect of plate oscillations was studied on the low Reynolds number instability in steady flow over finite thickness viscoelastic gels. The steady flow past a viscoelastic gel is known to exhibit a low Reynolds number instability, and the transition velocity has a minimum at finite wave number. A similar instability is encountered for the oscillatory flow past a viscoelastic gel, but the dependence of the transition velocity amplitude on the frequency was found to be complicated. At low Reynolds number, the velocity amplitude for an oscillatory flow was found to be lower than that for a steady flow at all wave numbers, and the transition amplitude was found to increase with frequency. However, at higher Reynolds numbers, the transition amplitude for an oscillatory flow was found to be lower than that for a steady flow for certain wave numbers, indicating a destabilizing effect. It was also found that wall oscillations could have a complicated effect on the instability at intermediate and high Reynolds numbers, because of the complicated and non-monotonic dependence of the interfacial mean velocity amplitude on the frequency. In the zero Reynolds number regime, the transition velocity amplitude for an oscillatory flow was found to depend strongly on the gel viscosity $\eta_{g}$. Experiments were carried out on the stability 
of an oscillatory flow past a viscoelastic gel in the zero Reynolds number limit, and the results were in agreement with the theoretical predictions.

The electrohydrodynamic instability at the interface between a Newtonian fluid and a viscoelastic solid was also studied. In this case, there is a shear induced instability due to the discontinuity in the strain rate at the surface, and an electrohydrodynamic instability due to the Maxwell stresses at the interface. It is found that these two instabilities are strongly interacting. The presence of flow destabilizes the electrohydrodynamic instability, and the applied potential destabilizes the shear induced instability.

\section{References}

Coward A V and Papageorgiou D T 1994 Stability of oscillatory two-phase couette flow. IMA J. Appl. Math. 53: 75

Coward A V and Renardy Y Y 1997 Small amplitude oscillatory forcing on two-layer plane channel flow. J. Fluid Mech. 334: 87-109

Davis S H 1976 The stability of time-periodic flows. Ann. Rev. Fluid Mech. 294: 57

Gambhire P and Thaokar R 2012 Role of conductivity in the electrohydrodynamic patterning of air-liquid interfaces. Phys. Rev. E 86(3): 036301

King M R, Leighton D T and McCready M J 1999 Stability of oscillatory two-phase couette flow: Theory and experiment. Phys. Fluids 4: 833-844

Ku D N 1997 Blood flow in artries. Ann. Rev. Fluid Mech. 29: 399-434

Kumaran V, Fredrickson G H and Pincus P 1994 Flow induced instability of the interface between a fluid and a gel at low reynolds number. J. Phys. II France 4: 893-904

Melcher J and Smith C 1968 Electrohydrodynamic charge relaxation and interfacial perpendicular field instability. Phys. Fluids 207: 778-790

Ramanan V, Kumar K A and Graham M 1999 Stability of viscoelastic shear flows subjected to steady or oscillatory transverse flow. J. Fluid Mech. 379: 255-277

Saville D A 1997 Electrohydrodynamics: The taylor-melcher leaky dielectric model. Ann. Rev. Fluid Mech. 29: $27-64$

Thaokar R and Kumaran V 2004 Stability of oscillatory flows past compliant surfaces. Eur. Phys. J. B 41(1): $135-145$

Von Kerckzek C and Davis S 1974 Linear stability theory of oscillatory stokes layers. J. Fluid Mech. 62: 753

Yih C-S 1968 Instability of unsteady flows or configurations part 1. instability of a horizontal liquid layer on an oscillating plate. J. Fluid Mech. 31: 737 